\title{
A Hierarchical Fuzzy-neural Multi-model Applied in Nonlinear Systems Identification and Control
}

\author{
Feng Ye \\ School of Physics \& Information Engineering \\ Jianghan University \\ Wuhan, China \\ yefenglj@yahoo.com.cn
}

\author{
Wei-min Qi \\ School of Physics \& Information Engineering \\ Jianghan University \\ Wuhan, China \\ qwmin@126.com
}

\begin{abstract}
The paper brings forward a hierarchical fuzzyneural multi-model with recurrent neural procedural consequent par for systems identification, states estimation and adaptive control of complex nonlinear plants. The parameters and states of the local recurrent neural network models are used for a local direct and indirect adaptive trajectory tracking control systems design. The designed local control laws are coordinated by a fuzzy rule-based control system. The upper level defuzzyfication is performed by a recurrent neural network. The applicability of the proposed intelligent control system is confirmed by simulation examples and by a DCmotor identification and control experimental results. Two main cases of a reference and plant output fuzzyfication are considered - a two membership functions without overlapping and a three membership functions with overlapping. In both cases a good convergent results are obtained.
\end{abstract}

Keywords-Fuzzy-neural hierarchical multi-model; Recurrent neural networks; Systems identification

\section{INTRODUCTION}

In the last decade, the computational intelligence(CI), including artificial neural networks(ANN) and fuzzy systems(FS) became a universal tool for many applications. Because of their approximation and learning capabilities [1], the ANNs have been widely employed to dynamic process modeling, identification, prediction and control [2,3] Mainly, two types of ANN models are used: feedforward (FFNN) or static and recurrent (RNN) or dynamic. The first type of ANN could be used to resolve dynamic tasks introducing external dynamic feedbacks. The second one possesses its own internal dynamics performed by its internal local feedbacks so to form memory neurons $[4,5]$. The application of the FFNN for modeling, identification and control of nonlinear dynamic plants caused some problems which could be summarized as follows: (1) The dynamic systems modeling usually is based on the nonlinear autoregressive moving average model which need some information of input/output model orders, and input and output tap-delays ought to be used. (2) The FFNN application for multi-input multi-output systems identification needs some relative order structural information. (3) The lack of universality in ANN architectures caused some difficulties in its learning and a Backpropagation (BP) through time learning algorithm needs to be used. (4) Most of the ANN-based models are nonparametric ones [3], and so, not applicable for an indirect adaptive control systems design.

The major disadvantage of all this approaches is that the identification ANN model applied is a nonparametric one that does not permit them to use the obtained information directly for control systems design objectives.

Similarly to the static ANNs, the fuzzy models could approximate static nonlinear plants where structural plant information is needed to extract the fuzzy rules [6,7]. The difference between them is that the ANN model are global models where training is performed on the entire pattern range and the FS models perform a fuzzy blending of local models space based on the partition of the input space. So the aim of the neuro-fuzzy (fuzzy-neural) model is to merge both ANN and FS approaches so to obtain fast adaptive models possessing learning [8]. The fuzzy-neural networks are capable of incorporating both numerical data and expert's knowledge, and describe them in the form of linguistic IF-THEN rules. During the last decade considerable research has been devoted towards developing recurrent neuro-fuzzy models. In reference [9] an ANFIS with external feedback is used as a neuro-fuzzy controller. Through BP learning, ANFIS is adapted to refine, or derive the fuzzy IF-THEN rules using system input-output data. Due to the recurrent neuro-fuzzy model with internal dynamics, the recurrent fuzzy rules introduced the feedback in the antecedent and the consequent part of the model $[10,11]$, which is in fact a computational procedure. Reference[12] use the hierarchical approach to reduce the number of IF-THEN rules. A promising approach of recurrent neuro-fuzzy systems with internal dynamics is the application of the Takagi-Sugeno (T-S) fuzzy rules with a static premise and a dynamic function consequent part [13].

So, the present paper proposed to extend the power of the fuzzy rules, using in its consequent part a learning procedure instead of dynamic nonlinear function and to organize the defuzzyfication part as a second RNN hierarchical level incorporated in a new hierarchical fuzzy-neural multi-model (HFNMM) architecture. The output of the upper level represents a filtered weighted sum of the outputs of the lower level RTNN models. The fuzzy-neural hierarchical multimodel proposed uses only three membership functions (positive, zero and negative), which combine the advantages of the RNNs with that of the fuzzy logic, simplifying the structure, augmenting the level of adaptation and decreasing the noise. Also we propose in the consequent part of the 
fuzzy rules to use a learning procedures or sequences of procedures which extend its computational capabilities incorporating identification and control features in the same architecture.

\section{RTNN MODEL AND CONTROL LAWS DESCRIPTION}

\section{A. Architecture and Learning of the RTNN}

The RTNN model is described by the following equations[14]:

$$
\begin{aligned}
& X(k+1)=J X(k)+B U(k) \\
& Z(k)=\Gamma[X(k)] \\
& Y(k)=\Phi[C Z(k)]
\end{aligned}
$$

Where $X(k)$ is an N-state vector, $U(k)$ is a M-input vector, $Y(k)$ is an L-output vector, $\mathrm{Z}(\mathrm{k})$ is an L-vectoroutput of the hidden layer, $\Gamma(\cdot), \Phi(\cdot)$ are vector-valued activation functions like saturation, sigmoid or hyperbolic tangent, which have compatible dimensions, $\mathrm{B}$ and $\mathrm{C}$ are weight input and output matrices with compatible dimensions and block structure, corresponding to the block structure of $\mathrm{J}$.

The RTNN topology has a linear time varying structure properties like controllability, observability, reachability and identifiability, which are considered in [15]. These properties of the RTNN structure signify that starting from the blockdiagonal matrix structure of $\mathrm{J}$, we can find a correspondence in the block structure of the matrices $\mathrm{B}$ and $\mathrm{C}$, that show us how to find out the ability of learning of this RTNN.

\section{B. Direct and Indirect Adaptive Neural Control Laws}

The block-diagram of the direct adaptive neural control system is given in Fig. 1.

The control scheme contains three RTNNs. The RTNN-1 is a plant identifier, learned by the identification error $E_{i}=Y_{d}-Y$, which estimates the state vector and the plant parameters. The RTNN-2 and RTNN-3 are feedback and feedforward neural controllers, both learned by the control error $E_{c}=R-Y_{d}$.

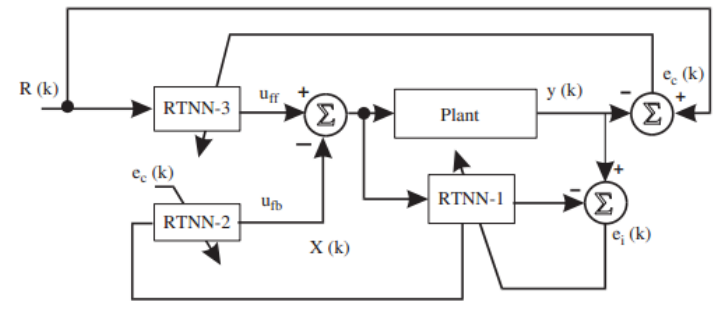

Figure 1. Block diagram of the direct adaptive RTNN control system

The block-diagram of the indirect adaptive neural control system is given in Fig. 2 [16,17].

The control scheme contains an RTNN identifier, learned by the same learning procedure, which issue a parameter and state information to the sliding mode controller, designed using the methodology given in [17]. The SM control law is given by the equations:

$$
\begin{aligned}
& U_{e q}(k)=(C B)^{-1}[-C J X(k)+R(k+1)+ \\
& \left.\sum_{i=1}^{p} \gamma_{i} E(k-i+1)\right] ;\left|\gamma_{i}\right|<1 \\
& U *(k)=\left\{\begin{array}{cc}
U_{e q}(k) & \text { if }\left\|U_{e q}(k)<U_{0}\right\| \\
-U_{0} U_{e q}(k) /\left\|U_{e q}(k)\right\| & \text { if }\left\|U_{e q}(k) \geq U_{0}\right\|
\end{array}\right.
\end{aligned}
$$

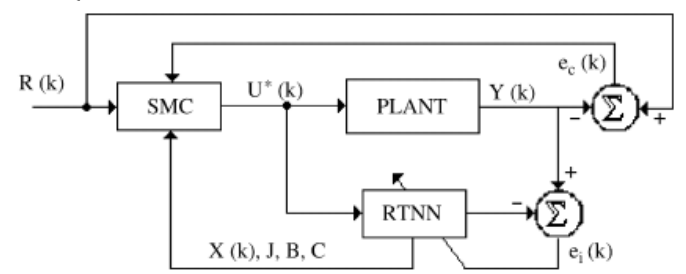

Figure 2. Block diagram of the indirect (SM) adaptive RTNN control system

Where $U 0$ is an upper bound of the control level, $p<N$ is the derivative order of the desired error model (sliding surface) and $\dot{\mathcal{i}}$ are parameters of the desired error model.

\section{HFNMM IDENTIFIER AND HFNMM CONTROLLERS DESCRIPTION}

\section{A. HFNMM Description}

Let us assume that the unknown system $y=f(x)$ generates the data $y(k)$ and $x(k)$ measured at $\mathrm{k}, \mathrm{k}-1, \ldots, \mathrm{p}$, then the aim is to use this data to construct a deterministic function $y=F(x)$ that can serve as a reasonable approximation of $y=f(x)$ in which the function $f(x)$ is unknown. The variable $\mathrm{x}$ is called an antecedent variable and the variable $\mathrm{y}$ is called a consequent variable. In FS modeling, the function $\mathrm{F}(\mathrm{x})$ is represented as a collection of IF-THEN fuzzy rules, represented by the statement:

IF antecedent proposition THEN consequent proposition.

The linguistic fuzzy model of Zadeh and Mamdani, cited in $[6,8]$ consists of rules $\mathrm{Ri}$, where both the antecedent and the consequent are fuzzy proposition:

Ri: If $x(k)$ is $A_{i}$ then $y(k)$ is $B_{i}, i=1,2, \ldots, P$

The model of T-S is a mixture between a linguistic and mathematical regression models, as it could be a dynamic state-space model, has a form as:

$\mathrm{R}_{\mathrm{i}}$ : if $\mathrm{x}(\mathrm{k})$ is $\mathrm{A}_{\mathrm{i}}$ and $\mathrm{u}(\mathrm{k})$ is $\mathrm{B}_{\mathrm{i}}$

$$
\text { Then }\left\{\begin{array}{c}
x_{i}(k+1)=J_{i} x_{i}(k)+B_{i} u(k) \\
y_{i}(k)=C_{i} x(k)
\end{array}\right.
$$

The output of the fuzzy neural multi-model system, represented by the upper hierarchical level of defuzzyfication is given by the following equation:

$$
Y(k)=\sum \omega_{i} y_{i}(k)
$$


Where $\omega_{i}$ are weights, obtained from the membership functions[18].

\section{B. Systems Identification by Means of HFNMM}

The systems identification is an essential part of the control systems theory and a powerful tool in the case when the plants mathematical model and their parameters are not known. In this point, the systems identification permits us to obtain plants structure, states and parameters when the output of the model follows the output of the plant. A blockdiagram of the dynamic systems identification, using an HFNMM identifier is given in Fig. 3.

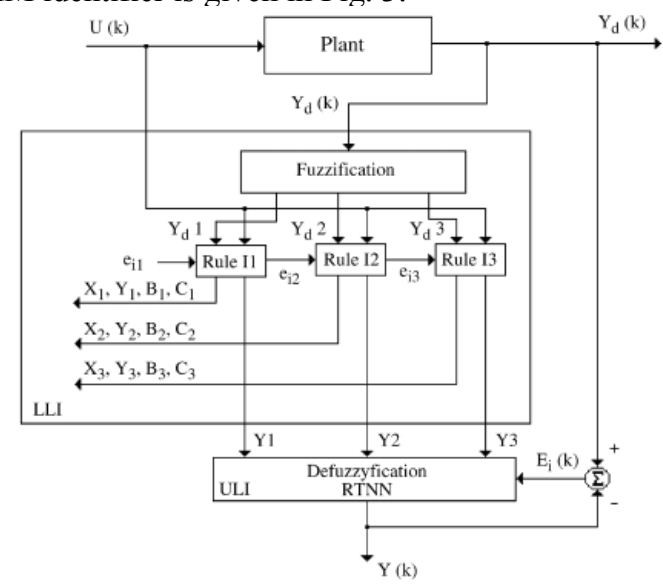

Figure 3. Detailed block diagram of the HFNMM identifier

\section{Direct and Indirect Adaptive Control by Means of HFNMM}

The structure of the entire identification system contains a Fuzzyfier, a fuzzy rule-based inference system (FRBIS), containing up to three $\mathrm{T}-\mathrm{S}$ rules and a defuzzyfier. The system uses an RTNN model as an adaptive, upper hierarchical level defuzzyfier. The local and global errors used to learn the respective RTNNs models are

$$
E_{i}(k)=Y_{d i}(k)-Y_{i}(k) ; E(k)=Y_{d}(k)-Y_{i}(k)
$$

The HFNMM identifier has two levels-lower hierarchical level of identification (LLI), and upper hierarchical level of identification (ULI). Detailed block diagram of the direct adaptive HFNMM controller is given in Fig. 4.

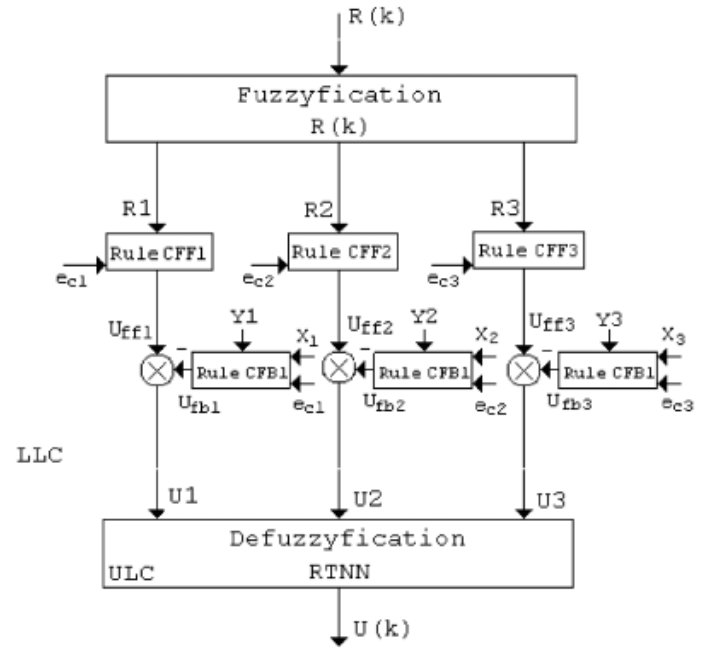

Figure 4. Detailed block diagram of the direct adaptive HFNMM controller

The detailed structure of the indirect adaptive HFNMM controller is given in Fig. 5. The identification part on the right contains three RTNNs, corresponding to the three rules, fired by the fuzzyfied plant output and taking part of the FRBIS HFNMM identifier, and the RTNN DF1 represents the defuzzyfier of the HFNMM identifier.

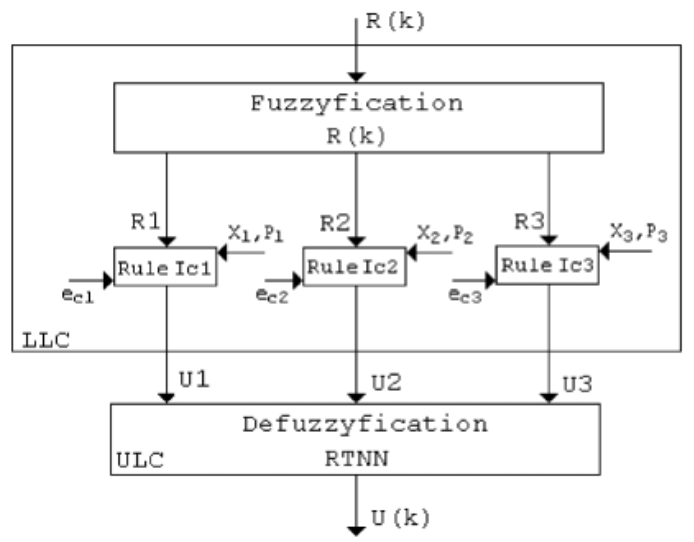

Figure 5. Detailed block diagram of the indirect adaptive HFNMM controller

\section{Simulation AND EXPERIMENTAL RESUltS}

Simulation results are given below as applying proposed tracking control methods for nonlinear plant model, then some experimental result of DC-motor control with the same direct HFNMM are given.

We use a nonlinear single-input single-output plant, given by the following equation:

$$
\begin{aligned}
y(k) & =0.972 y(k-1)+0.358 u(k-1)-0.129 u(k-2) \\
& -0.31 y(k-1) u(k-1)-0.042 y^{2}(k-2) \\
& -0.032 y^{2}(k-1) y(k-2)-0.35 y^{2}(k-1) u(k-2) \\
& +0.31 y(k-1) y(k-2) u(k-2)
\end{aligned}
$$


The graphical simulation results of the proposed indirect adaptive two RTNN model control, are given in Fig.6a-d.

The result, given in Fig. 6a shows that in the beginning, there are some discrepancy between the reference signal and the output plant signal due to improper identification, which reduces after few seconds, when the learning process has converged. The final MSE\% of control is about $2.5 \%$.
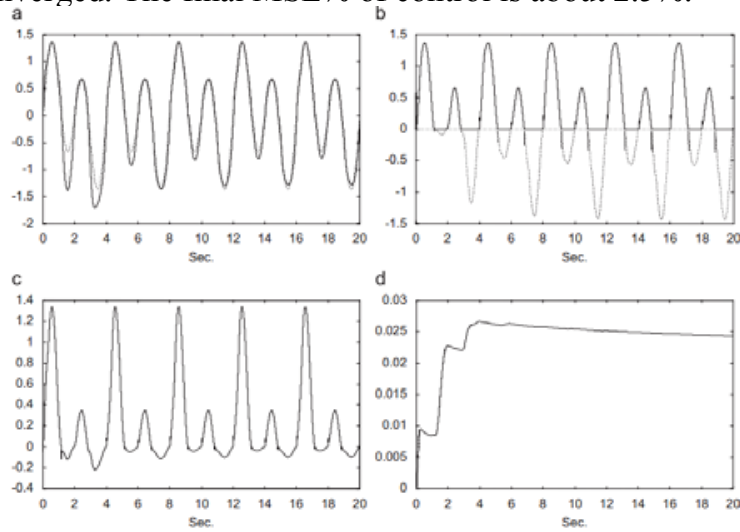

Figure 6. Graphical results of simulation using indirect adaptive fuzzy-neural multi-model control (example 1): (a) comparison of the output of the plant with the reference signal; (b) comparison of the output of the plant with the outputs of the identification RTNNs; (c) combined control signal and (d) mean squared error of control (MSE)

Fig.7a compares the output of the plant with the reference signal $r(k)=\sin (\pi k)+0.5 \sin (\pi k / 4)$, which is divided in two parts-positive and negative. The time of learning is 100s. The two identification and the two FF control RTNNs have topologies. Fig. $7 \mathrm{~b}$ shows the results of identification, where the output of the plant is divided in two parts, identified by two RTNNs. The state and parameter information issued by the identification multi-model is used to design a linear SM control law. The combined control signal and the MSE\% of control are given in Fig.7c,d. As it could be seen from the last graphics, the MSE\% of control are given in Fig.10c,d. As it could be seen from the last graphics, the $\mathrm{MSE} \%$ rapidly decreases, and reached values below $2 \%$
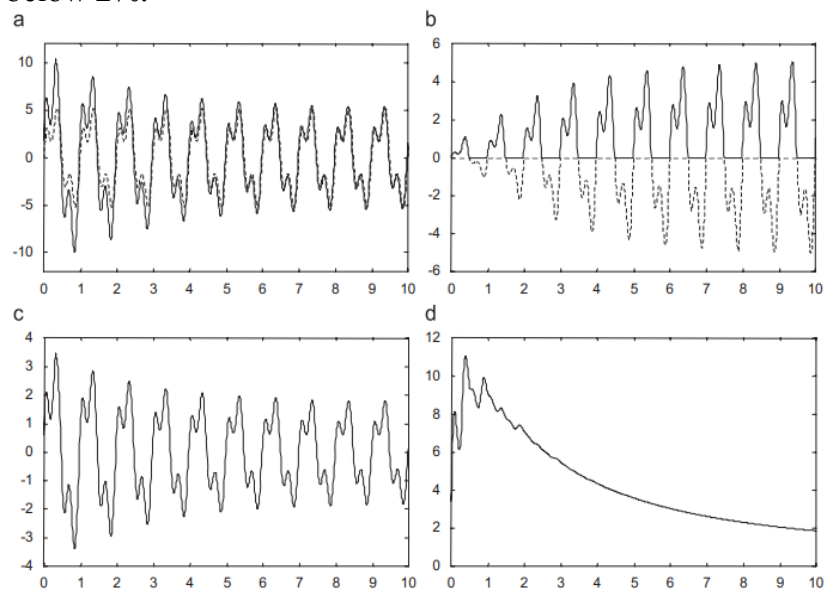

Figure 7.

Graphical results of simulation using indirect adaptive fuzzy-neural multi-model control (example 2): (a) comparison of the output of the plant and the reference signal; (b) comparison of the output of the plant and the outputs of the identification RTNNs; (c) combined control signal and (d)mean squared error of control(MSE\%)

Finally, a respective comparative results of direct adaptive plant control, obtained using only one RTNN, and that using HFNMM control are given in Fig. 8a-d, using the same 1-DOF mechanical plant model.
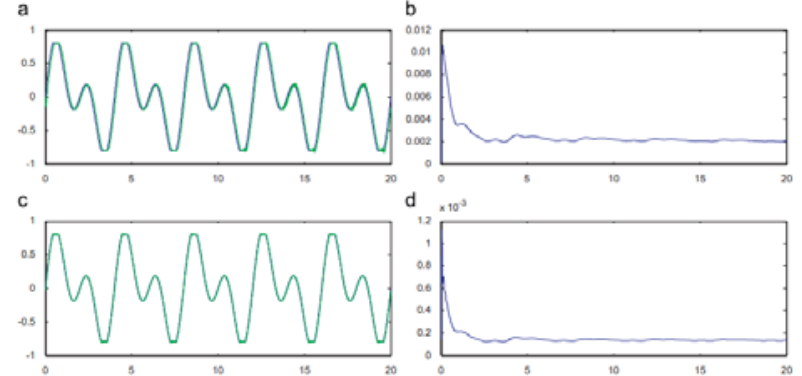

Figure 8. $\quad$ Comparative trajectory tracking control results obtained with: a single RTNN feedforward/feedback direct adaptive control, with an HFNMM feedforward/feedback direct adaptive control; and with a fuzzy control. (a) Comparison of the reference signal and the output of the plant using single RTNN controllers; (b) MSE of a single RTNN controller; (c) comparison of the reference signal and the output of the plant using HFNMM control, (d) MSE of an HFNMM control

From the graphics of Fig.8a-d, we could see that the direct adaptive HFNMM feedforward-feedback control is better than that using single RTNNs.

\section{CONCLUSIONS}

An HFNMM, containing fuzzy rules with procedural consequent parts, is proposed to be used for identification and control of complex nonlinear plants. Two control schemes (direct and indirect) using an HFNMM has been experimented and compared with a respective single-RTNN control and a fuzzy control. The proposed identification and control methods have the following advantages with respect to the conventional methods of intelligent fuzzy and neural control: (1) the number of T-S rules used is lower, (2) the level of adaptation is higher, (3) the resistance of noise and the robustness is superior due to the error correction (BP) learning of RTNNs applied.

\section{ACKNOWLEDGMENT}

This research is supported by key project of Wuhan science and technology bureau (Project Numbers: 201250499145-25)

\section{REFERENCES}

[1] S. Haykin, Neural Networks, "A Comprehensive Foundations", second ed., Prentice-Hall, Englewood Cliffs, NJ, 1999.

[2] K.J. Hunt, D. Sbarbaro, R. Zbikowski, P.J. Gawthrop, "Neural network for control systems (a survey)", Automatica 28,1992, pp.10831112

[3] K.S. Narendra, K. Parthasarathy, "Identification and control of dynamical systems using neural networks", IEEE Trans. Neural Networks 1 (1), 1990, pp.4-27. 
[4] P.S. Sastry, G. Santharam, K.P. Unnikrishnan, "Memory networks for identification and control of dynamical systems", IEEE Trans. Neural Networks 5 ,1994, pp.306-320.

[5] P. Frasconi, M. Gori, G. Soda, "Local feedback multilayered networks", Neural Compute. 4 ,1992, pp. 120-130.

[6] R. Babuska, "Fuzzy Modeling for Control", Kluwer, Norwell, MA, NY, 1998.

[7] C.T. Lin, C.S.G. Lee, "Neural Fuzzy Systems: A Neuro-Fuzzy Synergism to Intelligent Systems, Prentice-Hall", Englewood Cliffs, NJ, 1996.

[8] C.J. Lin, C.H. Chen, "Identification and prediction using recurrent compensatory neuro-fuzzy systems”, Fuzzy Sets and Systems 150 (2) ,2005, pp.307-330.

[9] J.S.R. Jang, "ANFIS: adaptive-network-based fuzzy inference system”, IEEE Trans. Systems Man Cybernet. 23 (3) ,1993, pp.665-685.

[10] J. Adamy, R. Kempf, "Regularity and chaos in recurrent fuzzy systems", Fuzzy Sets and Systems 140 (2) ,2003, pp.259-284.

[11] Y. Gao, M.J. Er, "NARMAX time series model prediction: feedforward and recurrent fuzzy neural network approaches", Fuzzy Sets and Systems 150 (2),2005, pp.331-350.
[12] A. Nürnberger, "A hierarchical recurrent neuro-fuzzy model for system identification”, Internat. J. Approx. Reason. 32 (2-3), 2003, pp.153-170.

[13] P.A. Mastorocostas, J.B. Theocharis, "A recurrent fuzzy-neural model for dynamic system identification”, IEEE Trans. Systems Man Cybernet. Part B: Cybernet. 32, 2002, pp.176-190.

[14] I. Baruch, E. Gortcheva, "Fuzzy neural model for nonlinear systems identification", in: Proc. AARTC IFAC Workshop, Cancun, Mexico, April 15-17, 1998, pp. 283-288.

[15] I. Baruch, J.M. Flores, F. Nava, I.R. Ramirez, B. Nenkova, “An advanced neural network topology and learning applied for identification and control of a D.C. motor", in: Proc. First Int. IEEE Symp. on Intelligent Systems, Varna, Bulgaria, September, 2002, pp. 289-295.

[16] I. Baruch, J.M. Flores, F. Thomas, R. Garrido, "Adaptive neural control of nonlinear systems", in: G. Dorffner, H. Bischof, K. Hornik (Eds.), Proc. Int. Conf. on NNs, ICANN 2001, Vienna, Austria, August 2001, Lecture Notes in Computer Science, Vol. 2130, Springer, Berlin, Heidelberg, NY, 2001, pp. 930-936.

[17] I. Baruch, L.A. Hernandez, J. Barrera-Cortes, "Adaptive discrete-time sliding mode control using recurrent neural networks", in: Proc. Second IFAC Symp. on System, Structure and Control, Oaxaca, Mexico, December 8-10, 2004. 